\title{
Haemoperitoneum from Spontaneous Rupture of Corpus Luteal Cyst in a Case of Dengue with Thrombocytopenia - A Rare Presentation
}

\author{
Mangala Chandrapal Raajput ${ }^{1}$, Shruti Shah ${ }^{2}$, Bhagyashree Priyadarshan Chitale ${ }^{3}$, \\ Eric John David ${ }^{4}$, Karthika Laxmi Dulam ${ }^{5}$, Mahija Venkata Sringaram6
}

\begin{abstract}
${ }^{1}$ Department of OBG, Wanless Mission Hospital, Miraj, Maharashtra, India. ${ }^{2}$ Department of OBG, Wanless Mission Hospital, Miraj, Maharashtra, India. ${ }^{3}$ Department of OBG, Wanless Mission Hospital, Miraj, Maharashtra, India. ${ }^{4}$ Department of OBG, Wanless Mission Hospital, Miraj, Maharashtra, India. ${ }^{5}$ Department of OBG, Wanless Mission Hospital, Miraj, Maharashtra, India. ${ }^{6}$ Department of Medicine, Wanless Mission Hospital, Miraj, Maharashtra, India.
\end{abstract}

\section{INTRODUCTION}

Dengue viruses are transmitted to humans by infected mosquitoes, mainly Aedes aegypti and albopictus. Dengue illness is clinically characterized by sudden onset of fever, intense headache, retro orbital pain, myalgia, maculopapular rash, minor bleeding manifestations like petechial rashes, gum bleeding, and severe haemorrhagic manifestations like haematemesis, epistaxis \& haemoperitoneum. Haemorrhagic manifestations can occur at any stage. Dengue shock syndrome is a dangerous complication with high mortality. ${ }^{1}$ Increased vascular permeability together with myocardial dysfunction, dehydration contributes to development of shock resulting in multi-organ failure like liver damage, neurological manifestation \& corpus luteal cyst rupture. Endothelial dysfunction induced by cytokines and chemical mediators, results in shock. Corpus luteum is a functional cyst developing in luteal phase of ovarian cycle, which regresses spontaneously to corpus albicans when pregnancy does not occur. Being a thin walled vascular structure corpus luteum is prone to haemorrhage even if bleeding is usually contained inside the cyst. Ruptured functional ovarian cyst is a frequent cause of acute pelvic pain in women of reproductive age. Invariably it is self-limiting with conservative management. ${ }^{2}$ Occasionally it requires surgical intervention, when accompanied by haemodynamic instability, large haemoperitoneum, especially in disturbed coagulation profile, as in our case.

Corpus luteal cyst rupture, with consequent haemoperitoneum is a rare disorder in women of reproductive age. The condition should be promptly diagnosed and treated because delayed diagnosis may significantly reduce women's fertility and lead to intra-abdominal bleeding, which may be life threatening. ${ }^{3}$ Ruptured corpus luteal cyst in some instances causes massive intra-peritoneal bleed leading to death, especially with bleeding diathesis. ${ }^{4}$
Corresponding Author:

Dr. Shruti Shah.

Wanless Mission Hospital, Miraj,

Maharashtra, India.

E-mail: s4shru@gmail.com

DOI: $10.14260 / \mathrm{jemds} / 2020 / 643$

How to Cite This Article:

Raajput MC, Shah S, Chitale BP, et al. Haemoperitoneum from spontaneous rupture of corpus luteal cyst in a case of dengue with thrombocytopenia - a rare presentation. J Evolution Med Dent Sci 2020;9(39):2935-2937, $10.14260 / \mathrm{jemds} / 2020 / 643$

Submission 23-12-2019,

Peer Review 13-08-2020

Acceptance 20-08-2020,

Published 28-09-2020.

Copyright (c) 2020 Mangala Chandrapal Raajput et al. This is an open access article distributed under Creative Commons Attribution License [Attribution 4.0 International (CC BY 4.0)] 


\section{PRESENTATION OF CASE}

A 21 years female, unmarried, resident of Malgaon, Miraj was referred from outside hospital to our Wanless Hospital, Miraj, on 16 / 09 / 2019, with chief complaints of high-grade fever with chills since last 3 days, along with pain abdomen, nausea $\&$ vomiting. There was no bleeding diathesis. Her previous menstrual history was regular. LMP was 3 weeks back. Her past history, family \& personal history was not significant.

On admission patient was conscious oriented, pallor +++, pulse 90 bpm, BP 110 / 70 mmHg, RS / CVS: NAD. P / A showed mild tenderness in lower abdomen. No P / S, P / V done as patient was unmarried. Outside investigations showed $\mathrm{Hb} 8.8$ gms, platelet count 15,000 cumm, TLC 8,900 / cumm, Dengue NS 1 reactive, Dengue IgG, IgM antibodies non-reactive, PT 17 sec, APTT $27 \mathrm{Sec}$, INR 1.18. Outside USG showed mild to moderate free fluid in peritoneal cavity with internal echoes, approximate $500 \mathrm{cc}$ haemoperitoneum. Patient was admitted in Medical critical ICU at Wanless Hospital. Patient was treated with $4 \odot$ RDP \& $1 \odot$ PCV.

Next day on 17 / 9 / 2019 investigations were repeated: $\mathrm{Hb} 7.3 \mathrm{gms}$, TLC 10,000 cumm, platelet count raised to 62,000 / cumm (due to IV $\odot$ RDP transfusion) PT $15 \mathrm{sec}$, APTT $30 \mathrm{sec}$, INR 1.28, TFT, LFT, RFT within normal limits, PS for MP -ve, Dengue serology (antibodies) -ve, Dengue NS 1 reactive, chest X ray - NAD, ECG-NAD. USG showed increased pelvic collection up to 1 to 1.5 liters with left haemorrhagic ovarian cyst about 4-5 cms. Right ovary, uterus, liver \& spleen normal. Aspiration revealed dark coloured blood suggestive of haemoperitoneum.

Patient was posted for exploratory laparotomy after taking on table death consent. On 17 / 9 / 2019, at 11 A.M., under GA, intraoperative revealed about 1.5 liters of haemoperitoneum which was aspirated. Left salpingo-oophorectomy was done as there was rupture of corpus luteal cyst which was intermittently bleeding.

After giving abdominal normal saline wash, and inspecting normal uterus and other side normal tube and ovary, abdomen was closed in layers. Intra-operative patient received $2 \odot \mathrm{PCV}, 10 \odot \mathrm{RDP}, 2 \odot \mathrm{FFP}$. Postoperative patient was shifted to Surgical ICU \& then ward. Patient tolerated the procedure well. POP was uneventful. Platelet count increased gradually. Patient was on antibiotics, corticosteroids (low dose). Investigations after discharge showed $\mathrm{Hb} 12.3 \mathrm{mg} / \mathrm{dL}$, TLC 13,700 cumm, platelet count 1, 57,000 cumm, HPR report showed haemorrhagic corpus luteal cyst. Patient was discharged on 27/9/2019 in good condition, after removal of suture and with a healthy wound.

Corpus luteal cyst rupture with consequent haemoperitoneum is a common disorder in women in the reproductive age. This condition should be promptly treated, as delayed diagnosis may significantly reduce women's fertility, and may lead to haemoperitoneum which may be life threatening. Ultrasound, contrast enhanced CT, MRI are imaging modalities available. 5

Acute pelvic pain in women of childbearing age is common $\&$ frequent cause for admissions to emergency room \& needs prompt evaluation. Sometimes it is difficult to distinguish from gastrointestinal \& urinary tract emergencies.

Most common cause of acute lower abdominal pain along with fainting attack with history of amenorrhoea is disturbed ectopic pregnancy, whereas other common cause is rupture of corpus luteal cyst leading to haemoperitoneum. Corpus luteal cyst haemorrhage may occur spontaneously or can be provoked by coital trauma, exercise.

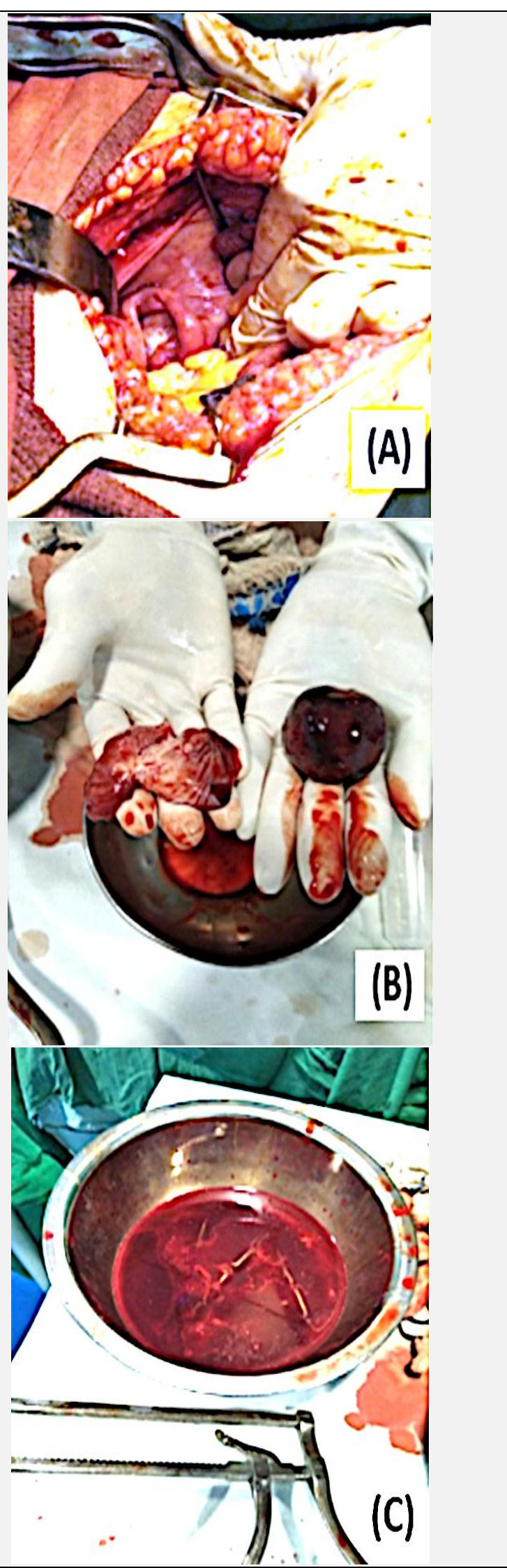

(A) Haemorrhagic Corpus Luteal Cyst on Left Side with Normal Tube and Ovary on Right Side, (B) Ruptured Corpus Luteal Cyst after Removal, (C) Part of Haemoperitoneum Which was Aspirated 
Corpus luteal cyst rupture leading to massive haemoperitoneum, progressively increasing, may prove fatal. The mortality is more when associated with coagulopathy, bleeding disorders, liver disorders idiopathic thrombocytopenic purpura and patients on anticoagulant therapy. ${ }^{6}$

In our case, due to low platelet count, probably due to dengue infection, there was progressive haemoperitoneum upto $1.5 \mathrm{~L}$ after rupture of a $5 \mathrm{~cm}$ corpus luteal cyst. After platelet replacement patient had undergone laparotomy with left salpingo- oophorectomy. Our hospital being a tertiary care centre \& Cardiothoracic (CTS) centre, we get lots of cases of cardiac valve replacement on anticoagulant therapy. We had 4 cases in last 5 years, of massive haemoperitoneum with corpus luteal cyst haemorrhage. In 3 cases wedge resection of ovary and suturing was done. All patients recovered and discharged from hospital. Incidence of corpus luteal cyst rupture is more in the right ovary. It is believed that dextro preponderance of corpus luteum rupture is probably due to presence of rectosigmoid colon protecting left ovary. Tang L.C. Cho HK, Chan has shown statistically significantly higher incidence and rupture on right side (81.25\%) as compared to left (56.52\%). They proposed hypothesis of difference in ovarian venous architecture that causes higher intraluminal pressure in right corpus luteum. ${ }^{7}$

\section{DISCUSSION}

Corpus luteum is a temporary hormone secreting remnant of mature ovarian follicle, after its rupture to release an ovum into the fallopian tube. Its main function is to secrete progesterone to maintain early pregnancy till placenta is developed by 8-10 weeks. If fertilization does not occur, corpus luteum involutes. Few days later, corpus albicans shrinks and menstruation occurs. However sometimes corpus luteum may fill with blood or other fluids, forming a cyst and rupture.

The corpus luteum is a highly vascular structure. The rate of blood flow to corpus luteum exceeds any other adult organ which is needed to deliver substrates for hormone production. This is also reflected by increased $\mathrm{O}_{2}$ consumption, 2-6 times that of liver. Corpus luteum are thin walled functional vascular structures and most of them are predisposed to rupture especially due to increased vascularity and rapid growth in luteal phase. This results in haemorrhagic cyst. Ongoing bleeding into cyst especially associated with coagulopathy, patients on anticoagulants results in rapid enlargement \& rupture leading to haemoperitoneum.

Acute pelvic pain in women of child bearing age is common and frequent cause for admission to emergency room requiring prompt medical evaluation especially in haemoperitoneum. The most common etiology of haemoperitoneum is ectopic pregnancy, ruptured corpus luteal cyst, endometriotic cyst rupture, ruptured hydropyosalpinx, ovarian torsion and acute appendicitis.

One should always keep differential diagnosis of corpus luteal cyst rupture in mind, especially in patients with bleeding disorders like Von-Willebrand disease, systemic anticoagulopathy disorder, sickle cell anemia, deranged coagulation, especially low platelets in Dengue, like our case.

Hallatt et al described the first large series of 173 patients with rupture corpus luteum cyst haemorrhage and haemoperitoneum. This entity can occur at all stages of women's reproductive life and is life threatening. Pregnancy increases risk of rupture with increased risk of abortion. ${ }^{8}$

\section{FINAL DIAGNOSIS}

Ruptured Corpus Luteal Cyst with Huge Haemoperitoneum with Early Dengue Haemorrhagic Shock Syndrome

Financial or Other Competing Interests: None.

\section{REFERENCES}

[1] Rajapakse S. Dengue shock. J Emerg Trauma Shock 2011;4(1):120-7.

[2] Pandit K, Potdar S, Pandit S, et al. Massive haemoperitoneum from a ruptured corpus luteal cyst. JOJ Case Stud 2018;5(5):555672.

[3] Aggarwal A, Goel P, Wauchu M, et al. Ruptured Corpus luteum with haemoperitoneum. J Obstetrics Gynecology Ind 2004;54(5):488-90.

[4] Baidya JL, Chakroborty J, Ray J, et al. Thrombocytopenia \& ruptured corpus luteal cyst: a deadly combination. J Med Dent Sci 2015;4(55):9674-76.

[5] Raziel A, Ron-EI R, Pansky M, et al. Current management of ruptured corpus luteum. Eur J Obstet Gynecol Reprod Biol 1993;50(1);77-81.

[6] Chandrashekhar NKT, Krishnappa R, Redddy CS, et al. Haemoperitoneum in dengue Fever with normal coagulation profile. J Glob Infect Dis 2013;5(1):29-30.

[7] Tang LC, Cho HK, Chan SY, et al. Dextropreponderance of corpus luteum rupture. A clinical study. J Reprod Med 1985;30(10):764-8.

[8] Hallatt JH, Steele CH, Snyder M. Ruptured corpus luteum with haemoperitoneum: a study of 173 surgical cases. Am J Obstet Gynecol 1984:149(1):5-9. 